\title{
HUMAN FACTORS ISSUES ASSOCIATED WITH LIMITED ABILITY AUTONOMOUS DRIVING SYSTEMS: DRIVERS' ALLOCATION OF VISUAL ATTENTION TO THE FORWARD ROADWAY
}

\author{
Robert E. Llaneras ${ }^{1}$, Jeremy Salinger ${ }^{2}$ \& Charles A. Green ${ }^{2}$ \\ ${ }^{1}$ Virginia Tech Transportation Institute \\ ${ }^{2}$ General Motors \\ Blacksburg, Virginia, USA \\ E-mail: ellaneras@vtti.vt.edu
}

\begin{abstract}
Summary: This study characterized driver behavior and established a foundation for defining functional performance requirements associated with a Limited Ability Autonomous Driving System (LAADS) - a system capable of automated steering and speed/headway maintenance tasks on freeways, but does not relieve drivers of all driving tasks. The research was designed to examine and reveal potential issues associated with the use of semi-autonomous systems, exploring impacts on willingness to engage in secondary non-driving related tasks, and driver allocation of visual attention while operating under LAADS (ACC and Lane Centering). Results found meaningful differences in the allocation of visual attention across ACC and LAADS driving under situations where drivers were engaged in a secondary task. Overall findings suggest that given a rudimentary, but reliable, LAADS system (one which does not monitor or otherwise restrict behavior) drivers are likely to increase the frequency of secondary task interactions, and engage in risky tasks that are likely to increase extended glances away from the forward roadway.
\end{abstract}

\section{INTRODUCTION}

Although the concept of a fully automated driving system as envisioned under the Automated Highway System program (Congress, 1994) has yet to be realized, technological advancements over the past decade have led to the emergence of advanced driver assistance systems and features such as Adaptive Cruise Control (ACC), collision warning, automatic braking, and lane keeping assist systems. To-date, deployed systems and features have largely been designed to support rather than replace the driver. Features like ACC, for example, enable drivers to relinquish partial control over the vehicle in order to increase convenience and safety through the automated management of longitudinal vehicle spacing. Crash avoidance technologies capable of automatically assuming limited control functions under defined situations (i.e., crash imminent braking) are also beginning to emerge into the market place, as are lane keeping assist systems that augment drivers by providing steering torque to aid drivers in maintaining their position within the lane. Advancements in driver assistance systems (e.g., adaptive cruise control and lane keeping assist) may be providing the early building blocks for future autonomous driving systems which assume full or partial authority from the driver. The deployment of these driverassist systems may lead to a wide range of operational concepts and transitions into autonomous driving, including Limited Ability Autonomous Driving Systems (LAADS) which may assume some control authority for steering and speed maintenance on limited-access freeways while still requiring drivers to monitor both the system as well as roadway and traffic environments. 
Substantive human factors challenges need to be addressed, however, before these forms of automated systems are to become a practical reality, and deliver on their expectations of increased safety and reduced human error. Issues include the potential for negative adaptations occurring through misunderstanding, misuse, over-reliance on the system, or changes in attention and distraction from the driving task. Another concern is how an automated system will impact drivers' information processing capabilities and level of workload, including their willingness to engage in non-driving related secondary tasks. Automation may also impact a driver's situational awareness, including their ability to perceive critical factors in the environment or detect system state changes (system failures) as their role shifts from active vehicle control to passive monitoring of the automated system and environment and path planning down the road. While drivers often have a choice of whether, when, and how long to take their attention away from the roadway, over-reliance on automation can influence these choices. Thus, the introduction of automation which controls vehicle speed maintenance, longitudinal and steering functions is likely, if not well designed or implemented, to further increase the frequency and nature of secondary task engagements as well as increase extended glances away from the forward roadway. This research was designed to examine and reveal potential issues associated with the use of semi-autonomous systems, exploring impacts on willingness to engage in secondary non-driving related tasks, and driver vigilance and allocation of visual attention while operating under a Limited Ability Autonomous Driving System (LAADS) capable of automated steering and speed/headway maintenance tasks on freeways, but does not relieve drivers of all driving tasks. The research reported here is part of a larger series of studies examining driver interactions with semi-autonomous driving systems; this paper focuses on influences of LAADS driving on driver secondary task interactions and allocation of visual attention.

\section{METHOD}

Evaluations were performed in a test track environment (GM Proving Grounds) using two 2009 Chevrolet Malibu test-bed vehicles, each equipped with a prototype Lane Centering (LC), and Adaptive Cruise Control (ACC) system. Test vehicles were instrumented with data collection and recording devices allowing vehicle, system, and driver interactions to be recorded during the test drives. The LAADS system required drivers to assume a supervisory role in order to monitor system states and the roadway environment for hazards. The system was limited in its operating environments and capabilities; it was not fully reliable, and required drivers to intervene with limited advance notice. The track study used a within-subjects, repeated measures design with 12 drivers to examine changes in driver behavior and visual attention demand when operating under semi-autonomous modes across a range of common freeway driving scenarios. A single independent variable (driving mode - ACC and LAADS driving) was experimentally manipulated. Participants completed two test drives experiencing both partial (ACC) and semiautonomous modes (LAADS consisting of ACC and Lane Centering) once familiarized with the vehicle and the test track environment. The order of these drives was counterbalanced across participants to control for order-based effects. This design allowed performance and behavioral changes under LAADS to be directly contrasted to ACC-only driving shown to be similar to manual driving (NHTSA, 2005), and provided an efficient means of discriminating driver's visual attention and ability to detect and respond to unexpected events under matched driving situations. Twelve individuals were recruited ranging from 25 to 34 years of age (average of 30 years of age), balanced by gender. Subjects were recruited from within the greater metropolitan 
Detroit, Michigan area and were screened over the phone with a verbal questionnaire to determine whether they met the study eligibility requirements. Individuals were compensated up to $\$ 80$ for their particpation.

\section{Procedure}

The driving session was separated into three phases: The familiarization phase acclimated drivers to the vehicle and test track environment over the course of a 15-minute drive wherein subjects drove the test vehicle manually, controlling the vehicle speed, longitudinal spacing and lane position. Following the familiarization phase, each subject completed two additional driving phases - an ACC and an Autonomous (LAADS) driving phase. The order of presentation for these phases varied across drivers with half receiving the ACC phase first followed by the LAADS driving phase. Driving scenarios exposed individuals to a range of common freeway traffic interactions and events (e.g., car following, passing, and system failures) and served to provide opportunities to characterize driver visual attention demand and responsiveness under ACC and LAADS. Primary measures emphasized indices related to driver allocation of visual attention and secondary task interactions. Subjective indices of performance were also captured via questionnaires intended to characterize driver perceptions associated with LAADS driving and system related performance aspects including: level of trust and comfort with the system, perceived vigilance and willingness to engage in secondary tasks.

Drivers were instructed that the purpose of the study was to evaluate two vehicle features during the session: an Adaptive Cruise Control system, and the LAADS system which was described as a Freeway Driving Assist system. Drivers were asked to pretend that these systems were commonplace and equipped on their personal vehicle and to imagine they were taking a long trip. Several techniques were used to set the stage to encourage and capture natural occurring secondary task interactions, including raising expectancy that the test drive would be boring and uneventful; making secondary tasks readily available (drivers were encouraged to bring their cell phone with them); providing access to reading material, food and drinks, CD's, etc.; and having the in-vehicle experimenter appear to mentally "check-out” by minimizing interactions with the driver and by informing participants that they had a homework assignment they needed to complete during the trip which involved interacting with a laptop and watching a DVD. This "fake homework" strategy proved to be very successful.

During the ACC driving phase, drivers were responsible for manually steering the vehicle with the ACC controlling speed and vehicle longitudinal spacing or headway. This phase served to provide comparative data to the LAADS drive. The ACC driving session took between 45 minutes and 1 hour, with drivers experiencing a range of driving scenarios. Drivers were free to engage in secondary task activities during this phase. During the LAADS driving phase, both ACC and Lane Centering functions were activated to enable autonomous driving. Each driving phase included a brief training and instruction segment which introduced drivers to the basic controls and displays associated with the ACC and Lane Centering systems. The LAADS driving phase lasted between 1.5 and 2 hours. Drivers were allowed to engage in secondary-tasks throughout the course of the drive without any prompting. At the completion of each driving phase, subjects were tasked with evaluating the system using a semantic differential scale and they completed a post-drive questionnaire and were then debriefed. 


\section{RESULTS}

Results provide strong evidence to suggest that when afforded the opportunity to relinquish vehicle speed maintenance and lane control functions to a rudimentary, but reliable autonomous system (one which does not monitor or otherwise restrict behavior), most drivers will engage in moderate to complex secondary task activities under the conditions examined. LAADS driving changed the propensity of secondary task engagements (increasing task engagements), as well as the nature of the task interactions leading to riskier behaviors (those requiring extended off-road glances) such as reading, reaching for object in back seat, and texting. Moreover, drivers tended to spend less time looking at the forward roadway, and had longer off-road glances when operating under the autonomous LAADS condition compared to non-autonomous driving.

\section{Secondary Task Engagements}

All drivers were observed to engage in some form of secondary task activity during the course of the approximately 3 hour drive while operating under both driving modes. Moreover, compelling evidence was found to suggest that drivers choose to engage in a broader range of tasks, including riskier activities, when operating under LAADS compared to ACC driving modes. As illustrated in Figure 1, drivers were found to engage in a variety of tasks, many commonly associated with driving (Dingus et al., 2006; Klauer et al., 2006), under both ACC and LAADS driving such as listening to music, conversing with the passenger, and interacting with the radio. However, riskier tasks such as reading, texting, and other phone/PDA interactions were almost exclusively limited to autonomous driving under Lane Centering.

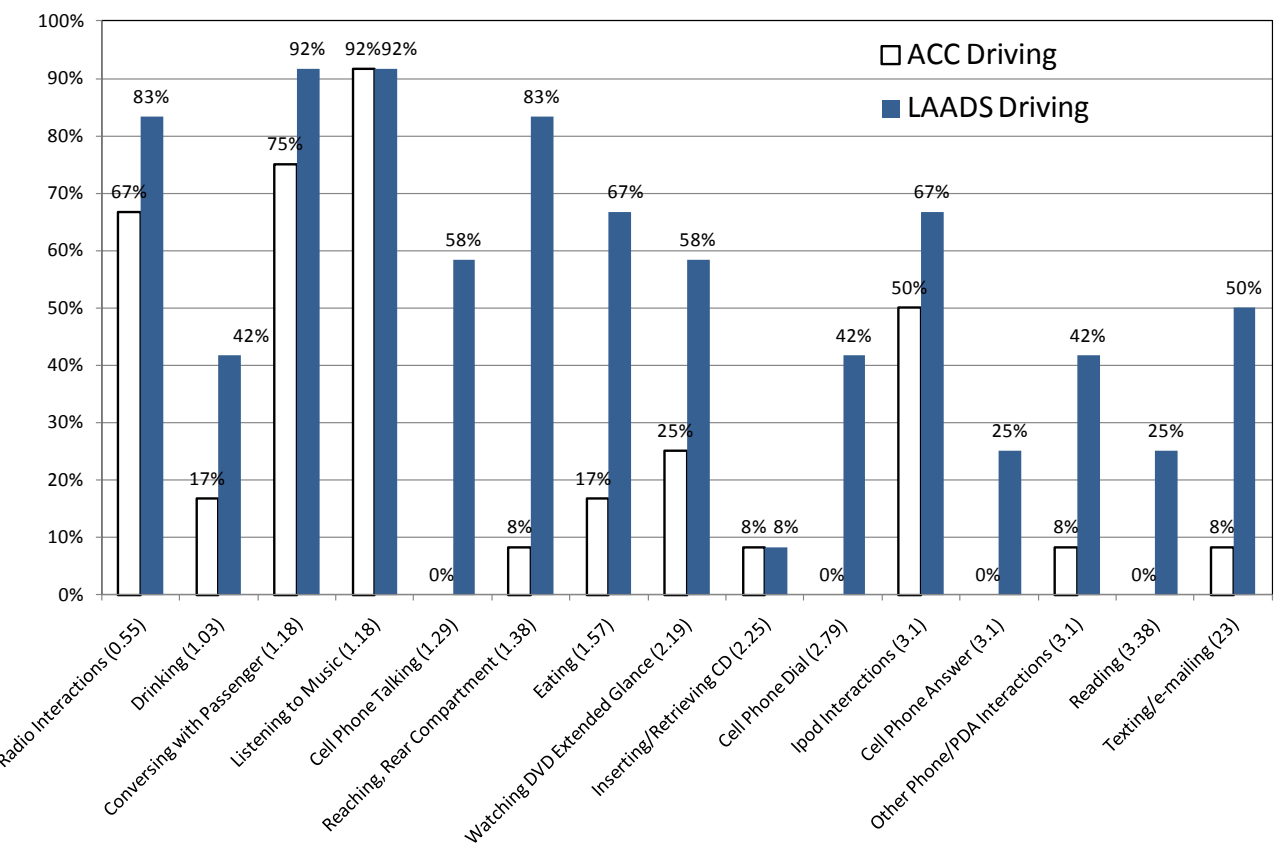

Figure 1 Percentage of Drivers Observed to Engage in Various Secondary Task Activities While Driving Under ACC and LAADS Modes (n=12). Tasks Presented in Order of Increasing Relative Risk Presented in the Parentheses Based on Klauer et al., 2006, and Hanowski et al., 2009

Drivers not only engaged in a wider range of tasks under LAADS, they also tended to perform some tasks more frequently relative to ACC driving. Examination of the mean task interaction 
frequencies (both raw and normalized by expressing frequencies as hourly interaction rates) across driving mode, revealed a general increase in some types of task interaction when operating under LAADS mode. Specifically, significant increases in task frequencies during LAADS driving were found for the following tasks: eating, reaching for an item in the rear compartment, dialing and talking on the cell phone, and texting/e-mailing (all these activities results in p values under 0.5). There was a widespread increase in the engagement in very risky tasks - those with relative risk values over 2.0, including watching DVD movies, reading, and texting (relative risk is an odds ratio expressing the likelihood of crash involvement relative to baseline non-distracted driving; a value of 1 indicates normal crash risk associated with baseline driving.).

\section{Off-Road Glances}

Overall, drivers were estimated to be looking away from the forward roadway approximately $33 \%$ of the time during the course of their 3 hour trip. Comparisons between ACC and LAADS head pose data reveal a significant increase in time spent looking away from the forward roadway under semi-autonomous relative to ACC-only driving $[F(11,1)=6.17, p=0.03]$. As shown in Figure 2, drivers tended to increase the percentage of time spent looking off-road by an average of 33 percent while driving under LAADS, suggesting that drivers were paying somewhat less attention to the forward roadway under the autonomous driving mode. This general finding is consistent with the secondary task data presented earlier suggesting that drivers engaged in more secondary activities under LAADS driving. Although this pattern was generally reliable, there were substantial individual differences in the magnitude of the effect across individuals with some drivers showing no increase under LAADS driving relative to ACC driving. Approximately one-third of the drivers (4 out of 12) showed substantial increases in the percentage of time spent looking off-road of at least 73\% when operating under LAADS.

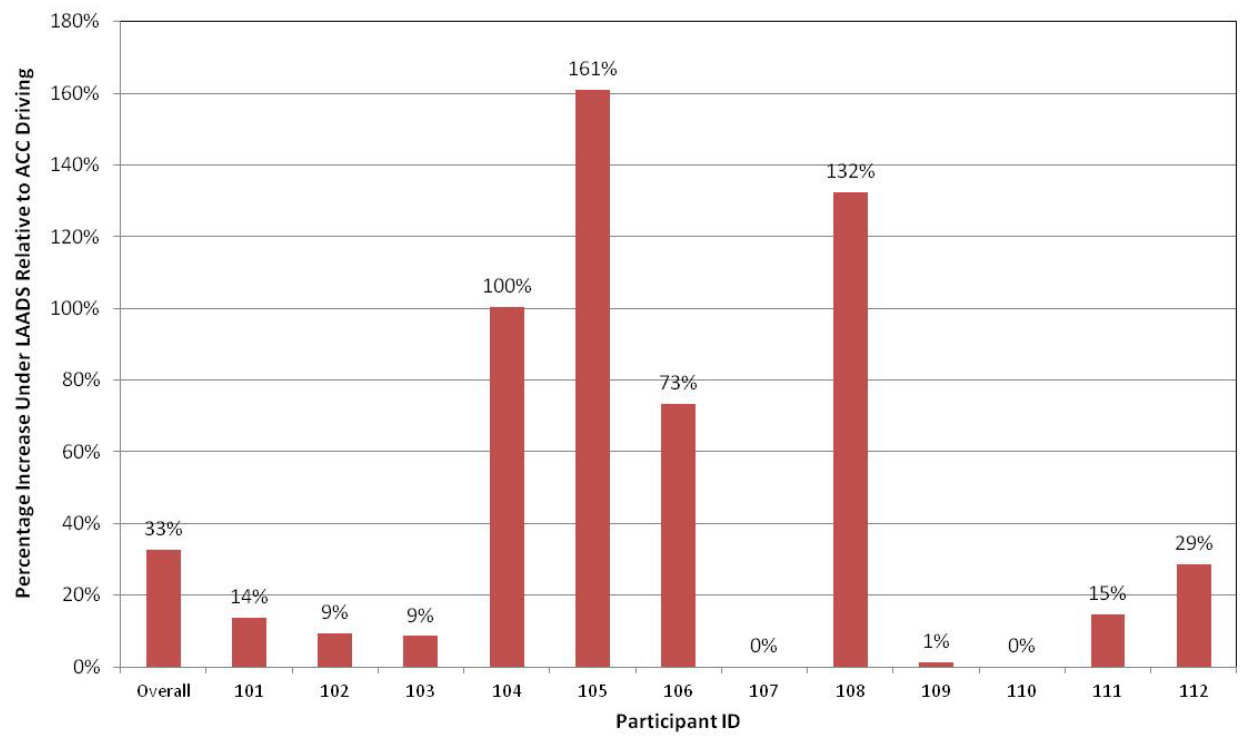

Figure 2 Overall Percentage Increase in Time Spent Looking Away from the Forward Roadway Under LAADS Driving Relative to ACC Driving 
Results generally found that looks away from the forward roadway tended to average less than 1 second (0.81 seconds), but were significantly influenced by driving mode. Drivers were found to have 26 percent longer off-road glance durations under LAADS compared to ACC driving, averaging $0.86 \mathrm{~s}$ and $0.68 \mathrm{~s}$ respectively $[\mathrm{F}(11,1)=46.99, \mathrm{p}<0.001]$. Although off-road looks extending beyond 2 seconds were relatively rare, occurring less than $8 \%$ of the time, drivers in our sample were estimated to have 3,325 off-road looks lasting greater than 2 seconds. The percentage of off-road looks greater than 2 seconds was found to increase by $27 \%$ under LAADS relative to ACC driving, with an average of $8.43 \%$ of glances versus $6.62 \%$ lasting over 2 seconds, respectively. A repeated measures ANOVA found that these differences were statistically significant $[F(11,1)=75.50, \mathrm{p}<0.001]$. Thus, drivers under LAADS were found to have, on average, significantly more off-road looks (looks away from the forward roadway) relative to ACC driving, and a significantly larger percentage of extended looks greater than 2 seconds in duration.

\section{SUMMARY \& CONCLUSIONS}

Drivers were observed to engage in a wide range of secondary task activities while operating under LAADS mode (ACC with Lane Centering) in which the responsibility for managing vehicle speed, spacing, and lane keeping functions were allocated to the vehicle. Moreover, riskier tasks (e.g., reading and texting) shown to increase crash risk under traditional driving (Klauer et al., 2006; Hanowski et al., 2009) were almost exclusively limited to autonomous driving mode. Reading, an activity estimated to increase crash risk by up to 3.4 times (Klauer et al., 2006), was performed by $25 \%$ of the sample in LAADS mode on the test track. Drivers not only engaged in a wide range of tasks under autonomous driving, the study also found changes in the interaction rates or frequency of occurrence for some tasks showing an increase in task interactions under LAADS relative to ACC driving. Drivers for example, increased their interaction rates for tasks such as eating, reaching for an item in the rear compartment, dialing and talking on the cell phone, and texting/e-mailing. While this trend was observed for drivers as a group, this study found large individual differences in regard to the nature and frequency of secondary task interactions suggesting that the impact of an autonomous system is not likely to be uniformly applied across all drivers.

Drivers signficantly increased the proportion of time spent looking away from the forward roadway under autonomous driving by an average of 33 percent relative to ACC driving. Analyses also examined the duration of individual "off-road glance" epochs where drivers were found to be looking away from the forward roadway. Since off-road glances extending beyond two seconds have generally been considered outside the established norms for safe driving (Weirwille, 1993; Zwahlen, 1988) and have been shown to increase crash risk (Dingus, et al., 2002), analyses quantified the proportion of “off-road glances” over 2 seconds occurring during both ACC and LAADS driving. LAADS was found to increase the duration of excessive offroad glances (those over 2 seconds in duration) by 27\%, on average; although this effect was not uniform across drivers, it was statistically signficant. Thus, drivers under LAADS conditions were found to have, on average, proportionately more extended glances away from the forward roadway relative to ACC-only driving. These data suggest that drivers may pay somewhat less attention to the forward roadway under LAADS relative to ACC-only driving; and that the primary means thru which this is accomplished is by increasing the opportunity for drivers to 
engage in secondary task activities. In summary, results suggests that given a rudimentary, but reliable, LAADS system (one which does not monitor or otherwise restrict behavior) drivers are likely to increase the frequency of secondary task interactions, and engage in tasks that are likely to increase extended glances away from the forward roadway. This work suggests that driving under a Limited-Ability Autonomous Driving System (LAADS) can potentially negatively impact the degree of visual attention drivers devote to the forward roadway (increases off-road glances). Results provide a foundation for the development and evaluation of countermeasure concepts designed to overcome or counter visual inattention problems and keep drivers focused on forward roadway, traffic environment, and maintain awareness of LAADS system state.

\section{ACKNOWLEDGMENTS}

This research was conducted by General Motors in partnership Virginia Tech Transportation Institute with support from the U.S. Department of Transportation Federal Highway Administration (DTFH61-08-H-00006). The authors would like to acknowledge the members of the research team including Mamatha Nanjundaiah, Shilpa Prabhuswamy at General Motors, Glenn Widmann and Robert Dufour at Delphi Electronics \& Safety, Sarah Koskie, and Nicola at IUPUI Driving Simulator Laboratory, Farhad Bolourchi at Nexteer Automotive, and Kunik Lee with FHWA.

\section{REFERENCES}

Congress, Anita (1994). The Automated Highway System: An idea whose time has come. Public Roads, Volume 58, Number 1.

Desmond, P. A., Hancock, P. A., and Monette, J. L. (1999). The impact of automation on driver performance and subjective state. In M. Scerbo and M. Mouloua (Eds.) Automation, Technology, and Human Performance: Current Research and Trends. (pp. 58- 62), Mahwah, NJ: Erlbaum.

Desmond, P., Hancock, P. A., and Monette, J. (1998). Fatigue and automation-induced impairments in simulated driving performance. Transportation Research Record, 1628, 8-14.

Dingus, T. A., Klauer, S. G., Neale, V. L., Petersen, A., Lee, S. E., Sudweeks, J., ... Knipling, R. R. (2006). The 100-car naturalistic driving study: Phase II - results of the 100-car field experiment (Report DOT-HS-810-593). Washington, D.C.: National Highway Traffic Safety Administration.

Endsley, M. R., and Kiris, E. O. (1995). The out-of-the-loop performance problem and level of control in automation. Human Factors, 37(2), 381-394.

Hancock, P. A., Dewing, W. L., and Parasuraman, R. (1993). A driver-centered system architecture for intelligent-vehicle highway systems. Ergonomics in Design, 2, 12-15, 35-39.

Klauer, S. G, Guo, F., Sudweeks, J., and Dingus, T. A. (2010). An analysis of driver inattention using a case-crossover approach on 100-car data (Report DOT-HS-811-334). Washington, D.C.: National Highway Traffic Safety Administration.

National Highway Traffic Safety Administration (2005). Automotive Collision Avoidance System Field Operational Test (ACAS FOT) Final Program Report. (Report DOT-HS-809-886) Washington, D.C.: National Highway Traffic Safety Administration.

Stutts, J. C., Feaganes, J., Rodgman, E., Hamlett, C., Meadows, T., Reinfurt, ... Staplin, L. (2003). Distractions in everyday driving. Washington, D.C.: AAA Foundation for Traffic Safety. 